\title{
Improving the Solidified Structure by Optimization of Coil Configuration in Pulsed Magneto-Oscillation
}

\author{
Jing Zhao ${ }^{1} \cdot \mathrm{Ji}-\mathrm{Hao} \mathrm{Yu}^{2} \cdot \mathrm{Ke} \mathrm{Han}^{3} \cdot$ Hong-Gang Zhong ${ }^{2} \cdot$ Ren-Xing $\mathrm{Li}^{2} \cdot \mathrm{Qi}-J i e \mathrm{Zhai}^{2}$
}

Received: 28 May 2018/Revised: 10 June 2018/Published online: 8 September 2018

(C) The Chinese Society for Metals and Springer-Verlag GmbH Germany, part of Springer Nature 2018

\begin{abstract}
Using both numerical and experimental methods, we studied the effect of coil configuration of pulsed magneto-oscillation (PMO) on distribution of electromagnetic field, flow field and solidification structure with the same pulse current parameters in $\mathrm{Al}$ ingots. We designed and constructed three types of coils: surface pulsed magneto-oscillation, hot-top pulsed magneto-oscillation (HPMO) and combined pulsed magneto-oscillation (CPMO). PMO treatment refined the solidification structure in all the ingots. The configuration of the PMO, however, introduced differences in magnetic field intensity, electromagnetic force, Joule heat, flow field, equiaxed grain zone, grain size and growth direction of columnar grains. The largest equiaxed grain zone was found in CPMO treated ingot, and the smallest grain size was found in both HPMO and CPMO treated ingots. Numerical simulation indicated that difference in electromagnetic field and flow field resulted in differences in solidification structure. HPMO is more advantageous over others for large ingot production.
\end{abstract}

Keywords Pulsed magneto-oscillation · Coil configuration · Electromagnetic field · Flow field · Solidification structure · Optimization

\section{Introduction}

Controlling the metal solidification process is one of the effective ways to refine solidification structure and improve the ingot properties [1]. In recent years, using electromagnetic field to modify solidification structure has attracted more and more attention because this technology is convenient and environmentally friendly [2-4]. Pulsed magneto-oscillation (PMO) is a newly developed metal solidification control technology after electric current pulse [5] and pulsed magnetic field [6]. This technology can significantly refine solidification structure and reduce ingot segregation. In PMO treating process, the melt would not

Available online at http://link.springer.com/journal/40195

Qi-Jie Zhai

qjzhai@shu.edu.cn

1 Department of Mechanical and Electrical Engineering, Tangshan University, Tangshan 063000, China

2 State Key Laboratory of Advanced Special Steel, Shanghai University, Shanghai 200444, China

3 National High Magnetic Field Laboratory, Florida State University, Tallahassee 32310, USA be contaminated and splashing would not occur at the top surface of the melt $[7,8]$. Gong et al. were able to refine the structure and reduce macro-segregation using PMO to cast $\mathrm{Al}$ and $\mathrm{Sn}-10.4 \% \mathrm{~Pb}$. Using an optimum value of pulse frequency and current intensity in PMO, they refined solidification structure of ferritic stainless steel [9]. Refinement occurs with PMO because the electromagnetic effect causes "crystal rain" falling from the top surface or mold wall, which increase the number of crystal nucleus [10]. After investigating the effect of pulse parameters, heat transfer condition of mold and geometry size on treatment effect with PMO, Yin et al. [11] attributed the refinement effect to both electromagnetic force and Joule heat generated by PMO. After studying the effect of pulsed parameters, pouring temperature, mold materials and preheating temperature on refinement, Li et al. [12] thought that temperature distribution was one of the major factors of nucleus survival in the process of nucleus falling. After investigating the PMO treatment in $\mathrm{Al}$ melt and measuring the temperature variation in the melt during solidification process [13]. Liang et al. [14] found that the thermal undercooling increased and the temperature gradient from the mold wall to the center of the casting decreased because of melt convection induced by the PMO treatment. 
Itzhak et al. studied the effect of application of PMO at different stages of solidification and under different cooling rate on both the solidification structure and the recalescence temperature [15-17]. Sun et al. [18] deduced that the convection in steel melt induced by PMO forced the grains to move from the solid-liquid interface, which resulted in the formation of uniformly distributed dendrites throughout the whole ingot. These researchers concluded that the refinement effect by PMO can be related to the material characteristics, heat transfer condition and parameters for generating pulsed field. These parameters changed the distribution of electromagnetic field and flow field. The effect of coil configuration in PMO on solidification structure, however, has not been studied systematically. In this work, we designed three types of PMO coil and studied the effect of coil configuration on the distribution of electromagnetic filed, flow field and solidification structure.

\section{Numerical Simulations and Experimental}

\subsection{Procedure}

Our work was divided into four tasks (Fig. 1). The first task was to design three coil configurations. The coils were put either on the top (named as SPMO), or around the hot-top (named as HPMO), or on and around the hot-top (named as CPMO) of the mold (Fig. 2). The second task was to simulate electromagnetic field and flow field in the melt under SPMO, HPMO and CPMO. The third task was to perform solidification experiments of $\mathrm{Al}$ under the same conditions as in our simulations. The last task was to analyze the relationship between field distributions and solidification structure so that the best PMO method could be determined.

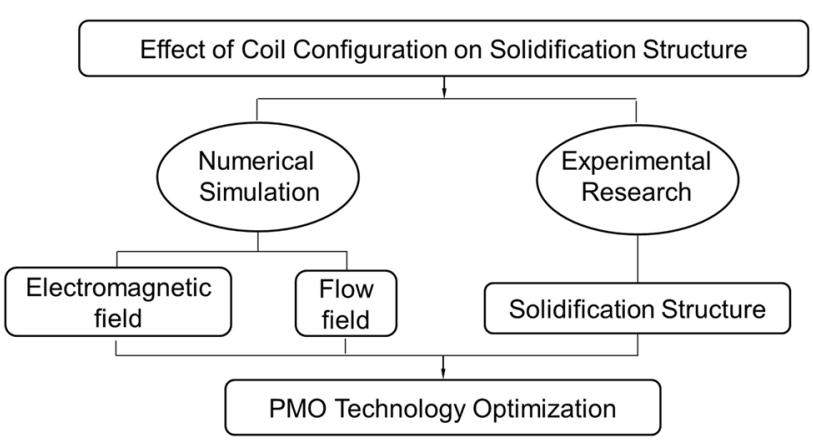

Fig. 1 Procedure of the study

\subsection{Numerical Simulation}

\subsubsection{Model}

Three finite element models were built according to coil configurations. Coil, melt, mold and air were included in the simulations. PLANE 13 (from ANSYS), which is a computational code for calculation of electromagnetic field and flow field, was used. Different grid sizes were chosen for different portion of the models. The finest grid was used in the melt (Fig. 3). To simplify mathematical model and save computation time, the calculations were based on five assumptions. (1) Al melt was considered as an incompressible conductor. (2) Current density in the coils remained uniform throughout the coil, which was determined by electric current, cross-sectional area of coil and windings. (3) No deformation occurred in the melt under electromagnetic force. (4) The systems were cylindrical and symmetric and could be simplified to two-dimensional models. (5) Limited space combined with unlimited boundary conditions was used to simulate the open magnetic field of air zone around the coil. The physical parameters of the $\mathrm{Al}$ are listed in Table 1. The main parameters of the pulse current used in the simulation include peak value and discharging frequency of the electric pulse. Typical values were $625 h_{I} \mathrm{~A}$ and $444 k_{t} \mathrm{~Hz}$, where $h_{I}$ and $k_{t}$ were the coefficients of the pulse generator. These values remained constant in the circuit.

\subsubsection{Governing Equations}

The governing Maxwell equations were used to simulate the electric magnetic field. They can be expressed as (1)(4):

$$
\begin{aligned}
& \nabla \times \boldsymbol{E}(r, t)=-\frac{\partial \boldsymbol{B}(r, t)}{\partial t}, \\
& \nabla \times \boldsymbol{B}(r, t)=\mu \boldsymbol{J}+\mu \varepsilon \frac{\partial \boldsymbol{E}(r, t)}{\partial t}, \\
& \nabla \cdot \boldsymbol{E}(r, t)=\frac{\rho}{\varepsilon} \\
& \nabla \cdot \boldsymbol{B}(r, t)=0
\end{aligned}
$$

where $\boldsymbol{E}$ is magnetic flux density vector, $\boldsymbol{B}$ is magnetic flux density vector, $\rho$ is electric charge density, $\boldsymbol{J}$ is total current density vector, $r$ is radius, $t$ is time, and $\varepsilon$ is permittivity.

The constitutive relations for the related electric fields are described in the following equations:

$\boldsymbol{J}=\sigma(\boldsymbol{E}+\boldsymbol{v} \times \boldsymbol{B})$,

$\boldsymbol{D}=\varepsilon \boldsymbol{E}$,

$\boldsymbol{B}=\mu_{0} \boldsymbol{H}$, 


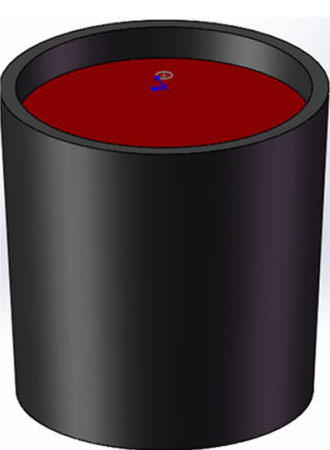

(a) Untreated

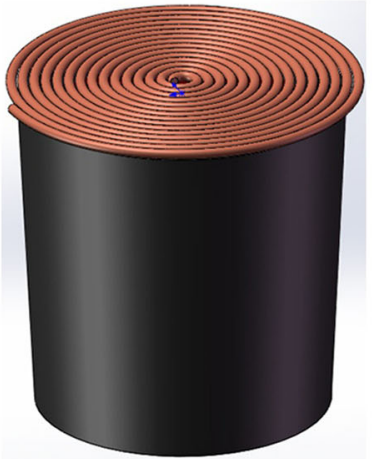

(b) SPMO

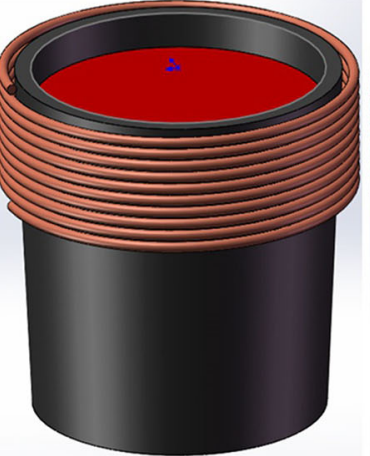

(c) HPMO

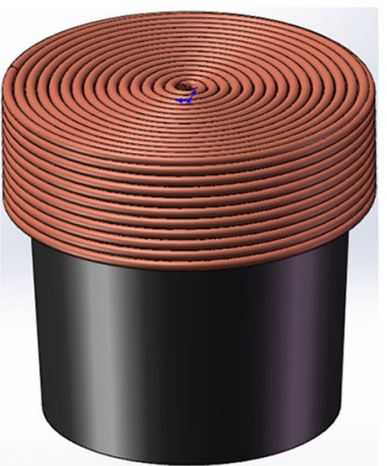

(d) CPMO

Fig. 2 Coil configurations of PMO

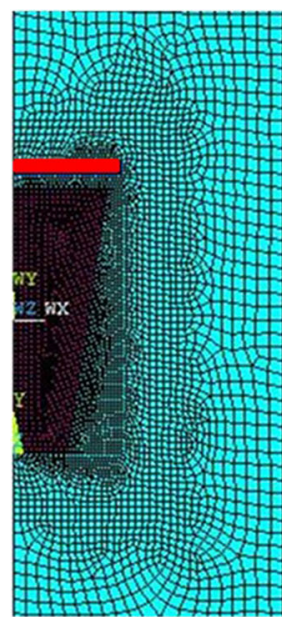

(a)

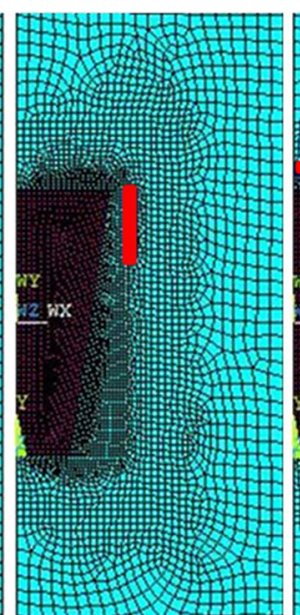

(b)

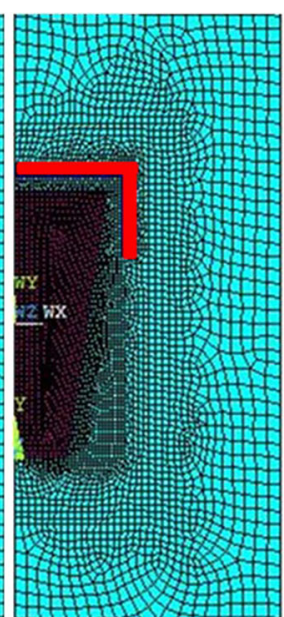

(c)

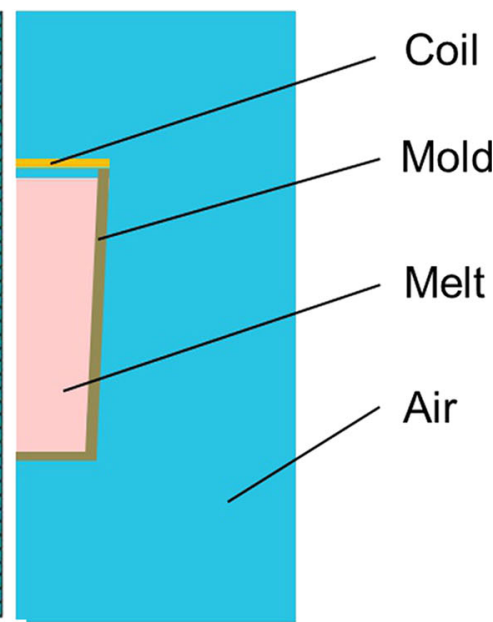

Coil

Mold

Melt

Fig. 3 FEM models used in the simulation: a SPMO; b HPMO; c CPMO

Table 1 Physical parameters of Al melt

\begin{tabular}{llll}
\hline Density $\left(\mathrm{kg} / \mathrm{m}^{3}\right)$ & Viscosity $(\mathrm{Pa} \mathrm{s})$ & Electrical resistivity $(\Omega \mathrm{m})$ & Relative permeability \\
\hline 2375 & 2.51 & $2.42 \times 10^{-7}$ & 1 \\
\hline
\end{tabular}

where $\boldsymbol{D}$ is electric flux density vector, $\sigma$ is conductivity, $\boldsymbol{v}$ is velocity vector, $\boldsymbol{H}$ is magnetic field intensity vector, and $\mu_{0}$ is magnetic permeability.

The pulsed electromagnetic field was induced by the pulsed electrical current in the coil. The current profile in the coil was similar to a sine wave, and the discharge time was much shorter than the charging time (Fig. 4). The relationship between the current and time was described as:

$I(t)=I_{0} \exp (-\zeta t) \sin (\omega t)$

where $I_{0}$ is a peak value in current, and $\zeta$ and $\omega$ are constant for the circuit.

In our simulation, one period was further divided into four stages for convenience. They are stages a $(T / 4), \mathrm{b}(T /$

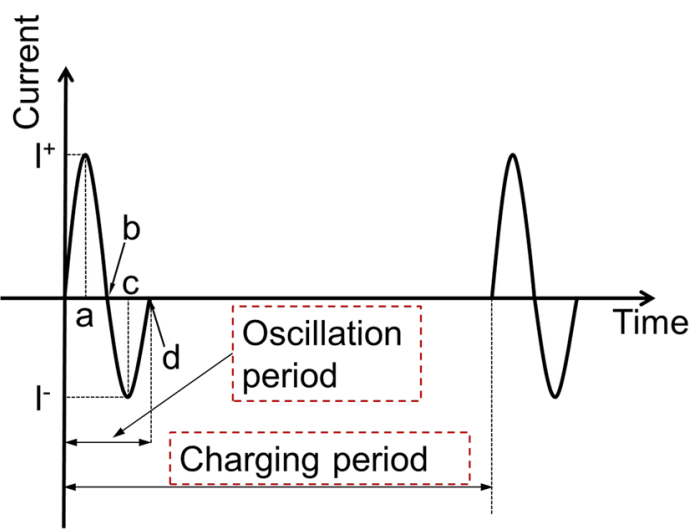

Fig. 4 Variation of pulsed electricity in a coil: a $1 / 4$ period; b $1 / 2$ period; c 3/4 period; $\mathbf{d} 1$ period 
2), c (3T/4) and d (1T), where $T$ is an oscillation period of PMO.

The continuity and the momentum equations in twodimensional model are described in Eqs. (9) and (10):

$\frac{\partial \boldsymbol{u}}{\partial \boldsymbol{x}}+\frac{\partial \boldsymbol{v}}{\partial \boldsymbol{y}}=0$,

$\boldsymbol{u} \frac{\partial \boldsymbol{u}}{\partial \boldsymbol{x}}+\boldsymbol{v} \frac{\partial \boldsymbol{u}}{\partial \boldsymbol{y}}=-\frac{1}{\rho} \frac{\partial \boldsymbol{P}}{\partial \boldsymbol{x}}+v\left(\frac{\partial^{2} \boldsymbol{u}}{\partial \boldsymbol{x}^{2}}+\frac{\partial^{2} \boldsymbol{u}}{\partial \boldsymbol{y}^{2}}\right)+\boldsymbol{F}_{\boldsymbol{x}}$

$\boldsymbol{u} \frac{\partial \boldsymbol{v}}{\partial \boldsymbol{x}}+\boldsymbol{v} \frac{\partial \boldsymbol{v}}{\partial \boldsymbol{y}}=-\frac{1}{\rho} \frac{\partial \boldsymbol{P}}{\partial \boldsymbol{y}}+v\left(\frac{\partial^{2} \boldsymbol{v}}{\partial \boldsymbol{x}^{2}}+\frac{\partial^{2} \boldsymbol{v}}{\partial \boldsymbol{y}^{2}}\right)+\boldsymbol{F}_{\boldsymbol{y}}$

where $\rho$ is density of the melt, $\boldsymbol{u}$ is velocity vector component in $\boldsymbol{x}$ axil direction, $\boldsymbol{v}$ is velocity vector components in $\boldsymbol{y}$ axil direction, $\boldsymbol{P}$ is pressure, $v$ is viscosity, $\boldsymbol{F}_{\boldsymbol{x}}$ is electromagnetic force vector component in $\boldsymbol{x}$ axil direction, $\boldsymbol{F}_{\boldsymbol{y}}$ is electromagnetic force vector component in $\boldsymbol{y}$ axil direction, which can be calculated according to the following equation:

$\boldsymbol{F}=\mu_{0} \boldsymbol{J} \times \boldsymbol{H}$.

The electromagnetic force, which induces force convection in melt, can be considered a momentum source imposed on the flow. To study this, the calculated electromagnetic force was used in flow field simulations.

\subsection{Experimental Method}

Commercial purity aluminum (Al: 99.7 wt $\%$, Fe: 0.2 wt $\%$, Si: $0.1 \mathrm{wt} \%$ ) was melted in a resistance furnace. After being held for $30 \mathrm{~min}$ at $750{ }^{\circ} \mathrm{C}$, the melt was poured into a graphite crucible with an inner dimension of $\Phi$ $140 \mathrm{~mm} \times$ height $200 \mathrm{~mm} \times$ wall thickness $10 \mathrm{~mm}$, preheated to $200{ }^{\circ} \mathrm{C}$ and PMO treated immediately. Four ingots were produced with the same process in the study. One ingot was untreated. Three other ingots were treated in a same solidification fixture under either SPMO, or HPMO, or CPMO with the same pulsed parameters (Fig. 5). The

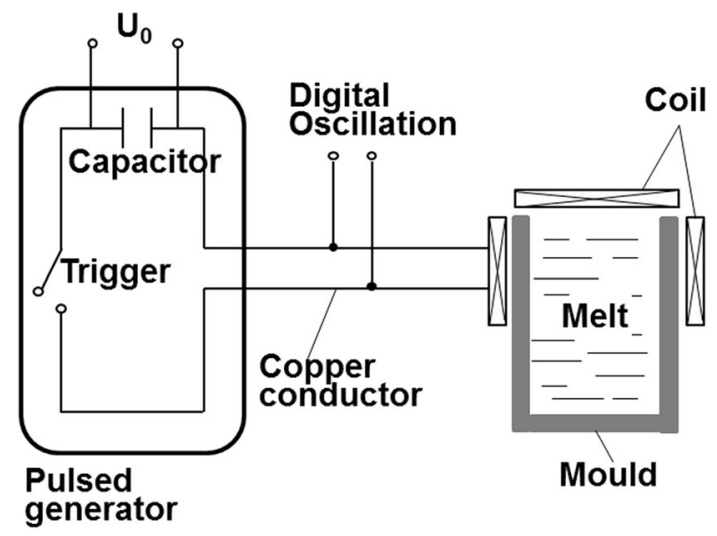

Fig. 5 Schematic illustration of experimental setup peak value of pulse current was $625 h_{I}$ A with a pulse frequency of $444 k_{t} \mathrm{~Hz}$, which were identical to those used in numerical simulation. The ingots were cut along the central longitudinal section and then etched for metallographic examination. The etchant was a mixture of 3 hydrochloric acid: 3 nitric acid: 5 water. Specimens in the lower part of ingots (Fig. 6b) were mechanically polished by vibration polisher for $3 \mathrm{~h}$ and then electronically polished with a mixture of distilled water and fluoboric acid at $0.3-0.5 \mathrm{~A} /$ $\mathrm{cm}^{2}$ for $20-40 \mathrm{~s}$. The images were taken in a Zeiss light microscope (Axio Imager A2m).

\section{Simulation Results}

\subsection{Distribution of the Magnetic Field Intensity}

The distribution maps of magnetic field intensity in $\mathrm{Al}$ melt under SPMO, HPMO and CPMO at four stages in a period show that the direction, value and affected-zone in melt varied with the variation of pulse current (Fig. 7). Because of skin effect of an alternating electromagnetic field, induced magnetic field distributed mainly near the surface and mold wall. In the center of the melt, intensity was nearly zero. For the melt solidified under SPMO, the induced magnetic field distributed mainly in a narrow region near the top surface. For the melt solidified under HPMO and CPMO, however, the induced magnetic field distributed in a wider region, which approached to the interior of melt treated. Comparison of the value of magnetic field intensity indicated that the largest value appeared in CPMO treated melt (the maximum value close to 0.06 Tesla), which was twice of that in SPMO case, which has the smallest value of 0.03 Tesla. In all the configurations, the maximum moment occurred at $T / 4$

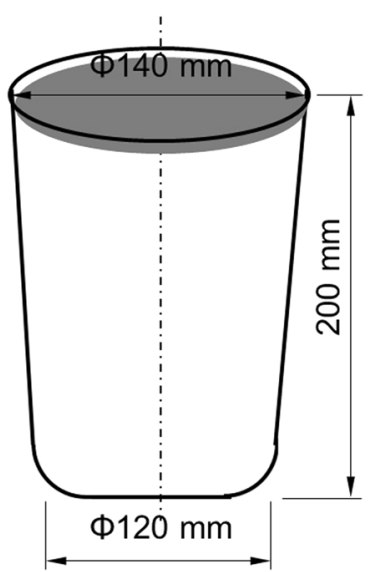

(a)

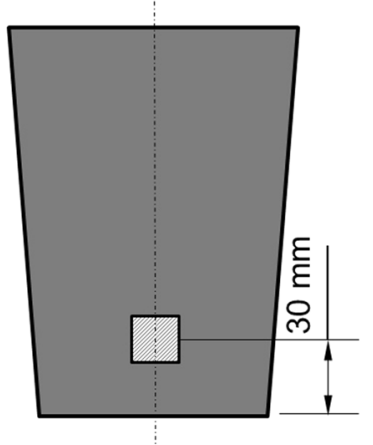

(b)
Fig. 6 Geometry of the ingot (a) and sampling instruction (b). $U_{0}$ is the discharge voltage 
(a)

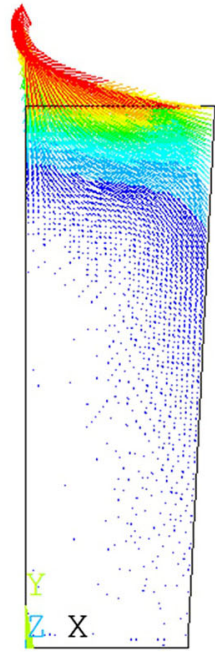

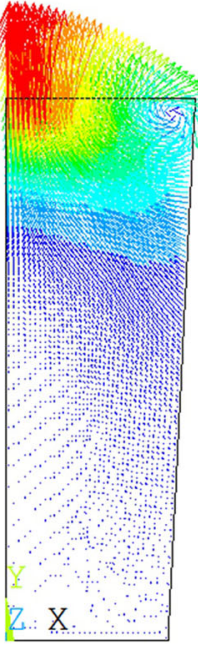

$\mathrm{T} / 2$

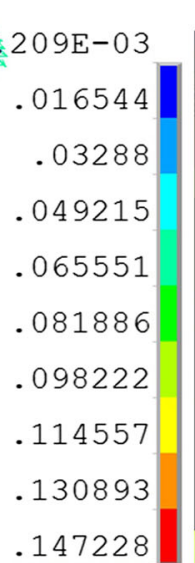

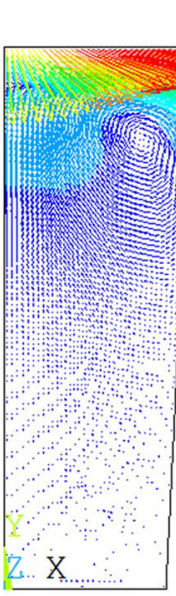

$871 \mathrm{E}-04$ .007121 .014156 .02119 .028224 .035258 .042293 .049327 .056361 .063395

\section{T/4}

(b)

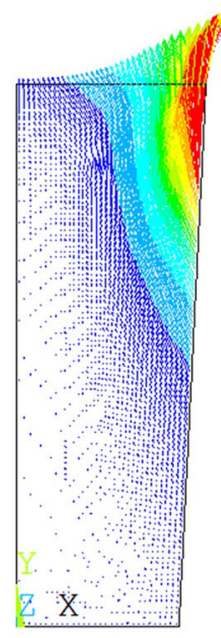

$\mathrm{T} / 4$

\begin{abstract}
$.885 \mathrm{E}-04$
.039125
.078161
.117198
.156234
.195271
.234307
.273343
.31238
.351416
\end{abstract}

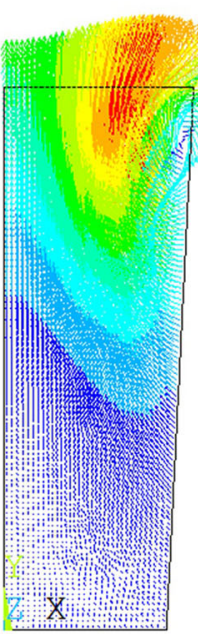

$3 T / 4$

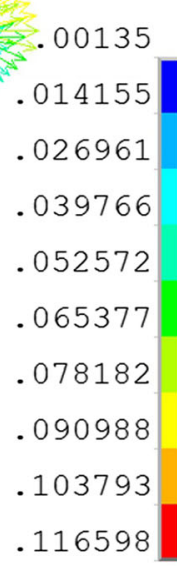

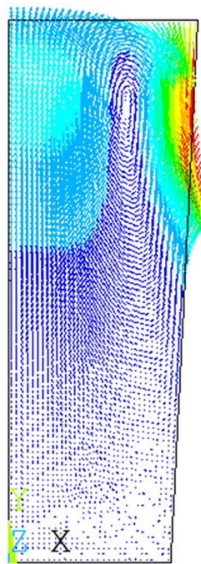

$\mathrm{T} / 2$

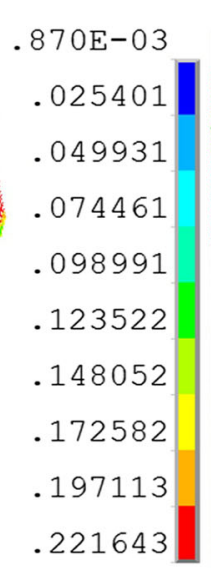

T

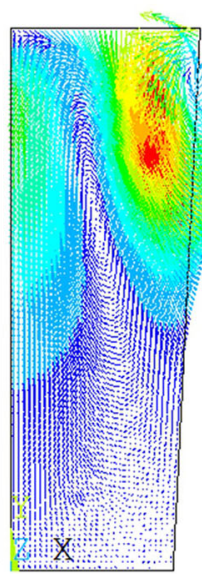

$.269 E-03$

.006545

.012821

.019097

.025372

.031648

.037924

.0442

.050475

.056751

(c)

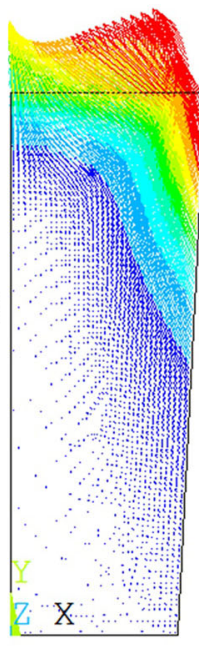

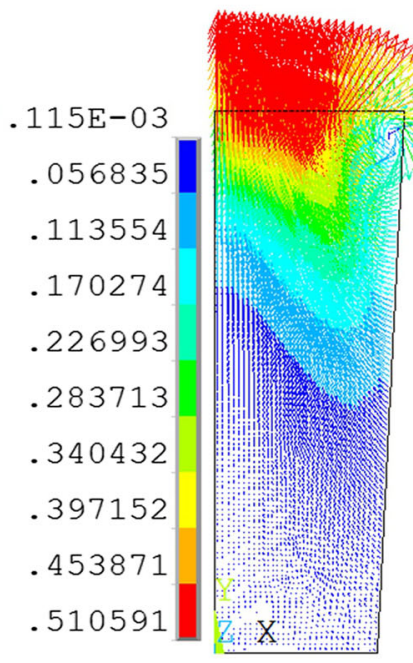

$\mathrm{T} / 4$

$\mathrm{T} / 2$

3T/4

T

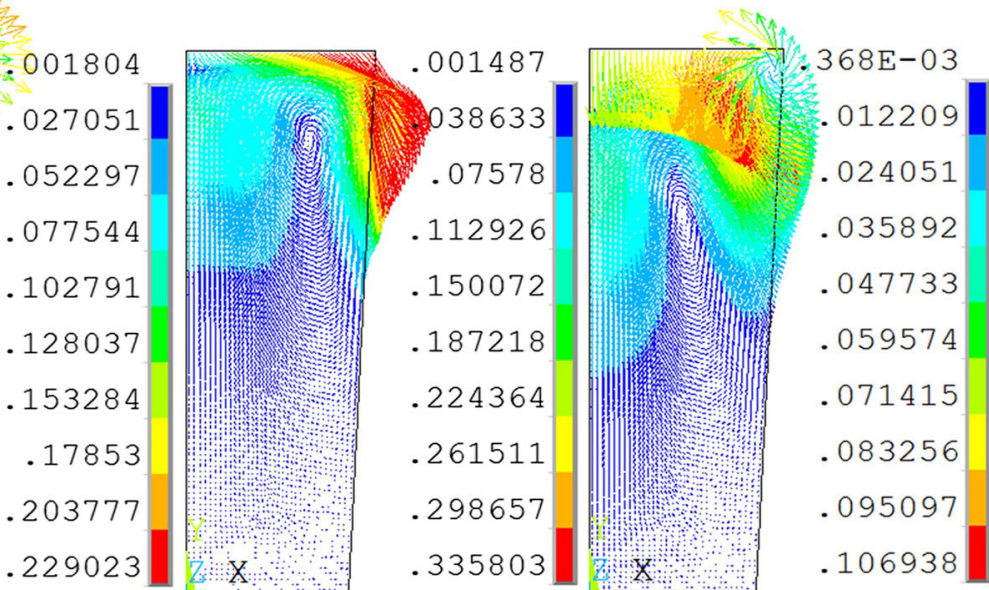

3T/4

Fig. 7 Distribution of the magnetic field intensity: a SPMO; b HPMO; c CPMO 
moment. ( $T$ is an oscillation period.) Comparison of the location demonstrated that the maximum value of magnetic field intensity for SPMO configuration appeared at the top surface and near the wall in a direction of at a small angle to the top surface. The maximum value with HPMO appeared at the half height of the coil in a direction of nearly vertical to the top surface. The magnetic field intensity in CPMO treated melt was the largest with an angle to the top surface and the wall. Therefore, the distribution, maximum value and direction were all related to the coil configuration.

\subsection{Distribution of Electromagnetic Force}

The variation of electromagnetic force under PMO with respect to time and space was similar that of magnetic field intensity (Fig. 8). The design of PMO coil governed the maximum value, the direction and the distribution of electromagnetic force. A comparing the electromagnetic force at $T / 4$ moment ( $T$ is an oscillation period) can be used to show the effect of the design of PMO coil on the maximum value, the direction and the distribution of electromagnetic force. In SPMO treated melt, the maximum electromagnetic force appeared at the top surface and near the wall with the direction of near parallel to the wall. In the HPMO treated melt, the maximum electromagnetic force located at the half height of coil with the direction perpendicular to the wall. In the CPMO treated melt, the maximum electromagnetic force was in a direction with an angle to the top surface and wall, and was the largest among three designed configurations.

\subsection{Distribution of Joule Heat}

Previous simulation results showed that Joule heat varied with time and space and the maximum of value of Joule heat appeared at $T / 2$ [19]. Distribution analyses of Joule heat at $T / 2$ in SPMO, HPMO and CPMO with the same pulsed current parameters showed that Joule heat was concentrated near the coil (Fig. 9). The maximum values of Joule heat in SPMO and HPMO treated melt were similar but at different locations. The CPMO generated the largest value of heat.

\subsection{Flow Field Distribution}

The analyses of the flow field induced by PMO treatment indicated that electromagnetic force mainly affected the melt in the upper part (Fig. 10). Two vortexes emerged in all PMO treated melts. The difference, however, was found on the relative location of two vortexes and the maximum value of velocity. Two vortexes were located horizontally near the top surface with the maximum value of velocity near the center and the wall in SPMO treated melt. Two vortexes were located near vertically in HPMO and CPMO treated melt, but the vortexes and the maximum value of velocity were larger in CPMO than in HPMO. The maximum velocity appeared near the wall under HPMO and in the center under CPMO.

\section{Experimental Results}

Macrographs reveal the structure of $\mathrm{Al}$ ingot solidified without and with SPMO, HPMO and CPMO (Fig. 11). Without PMO, the ingot consisted mainly of columnar grains with a small number of coarse equiaxed grains at the bottom of the ingot. PMO refined equiaxed grains and increased the equiaxed zone from $2.5 \mathrm{vol} \%$ (untreated) to $37.1 \mathrm{vol} \%$ (SPMO treated), $41.5 \mathrm{vol} \%$ (HPMO treated) and 81.3 vol\% (CPMO treated) (Table 2). The equiaxed zones with different size and shape were all at the bottom of the ingots in SPMO and HPMO ingots. The equiaxed zone in CPMO ingot extended from the bottom nearly to the top surface. Micrographs taken at the lower half of the ingots were used to estimate average size (Fig. 12, Table 2). The equiaxed grains were the coarsest in untreated ingot and the finest in HPMO and CPMO ingot. Previous researchers have shown that nucleation begins at the surface and near the mold wall because of the high cooling rates in these regions. Newly formed nuclei can separate from the solidified materials, drift to the interior of the mold and sink to the bottom of the mold because of the gravity. Without PMO in our experiment, gravity alone could not make grains sink effectively and fewer nuclei drifted down. Consequently, nuclei had room to coarsen and therefore equiaxed grains had large size. With PMO, sinking was assisted by electromagnetic force in the same direction as the gravity, resulting in more nuclei drifting into the interior of the melt. These drifted crystals, which were called "crystallization rain," increased the numbers of nuclei in the melt [20]. Finally, a fine-grained equiaxed zone formed at the bottom of the ingot. Thus, PMO effectively increased the equiaxed zone and decreased the size of grains.

PMO also changes the structure of upper columnar grains. PMO refined columnar grains and caused columnar grains to grow in multiple angles with respect to the wall of the mold. At the bottom of ingots, some columnar grains were found solidified without any PMO and with SPMO. Their growth direction was perpendicular to the mold bottom wall with a length of $18 \mathrm{~mm}$ for ingot without PMO and $13 \mathrm{~mm}$ for ingot with SPMO. This may be related to the cooling profile in the bottom of ingots. However, no columnar grain was found at the bottom of HPMO and CPMO ingots with the same cooling condition as SPMO. 
(a)

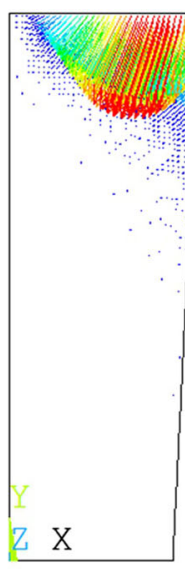

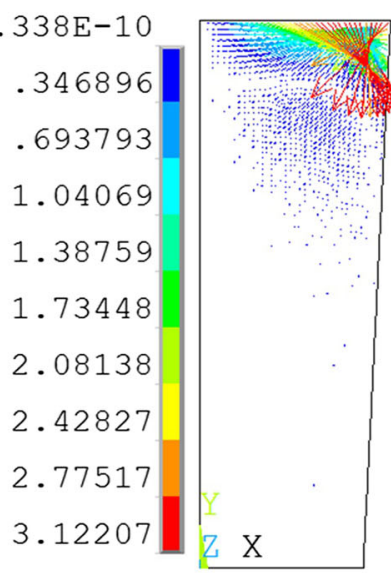

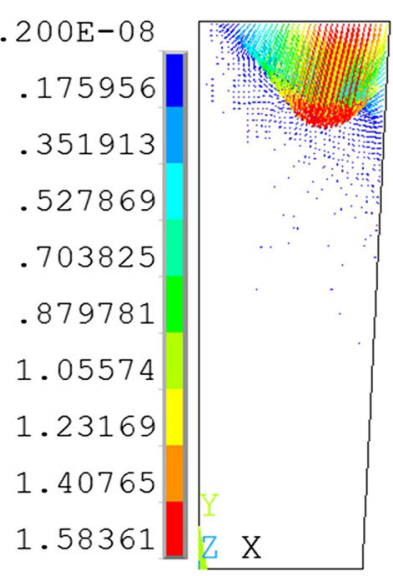

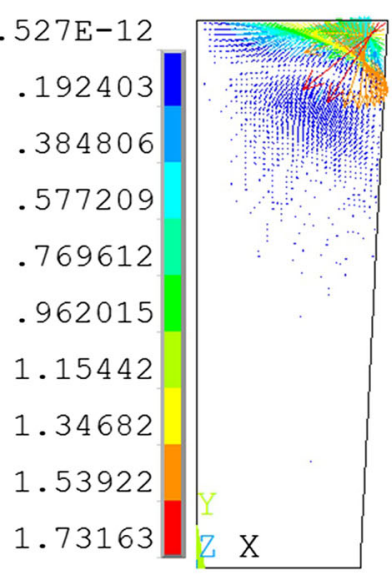

$.252 E-09$

.047634

095267

.142901

.190535

.238169

.285802

.333436

.38107

.428704

$\mathrm{T} / 4$

$\mathrm{T} / 2$

$3 T / 4$

$\mathrm{T}$
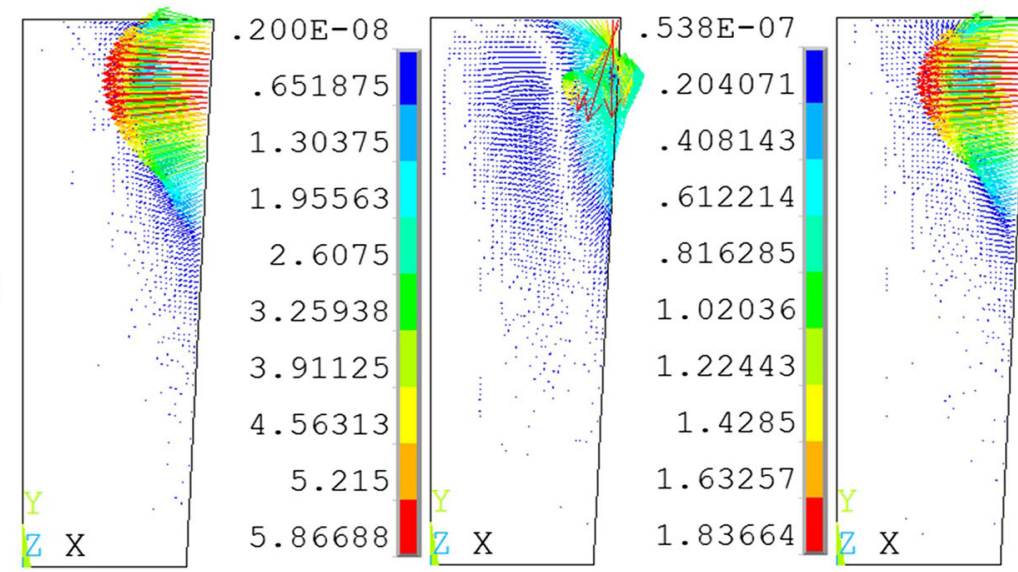

$.457 \mathrm{E}-08$
.351181
.702363
1.05354
1.40473
1.75591
2.10709
2.45827
2.80945
3.16063

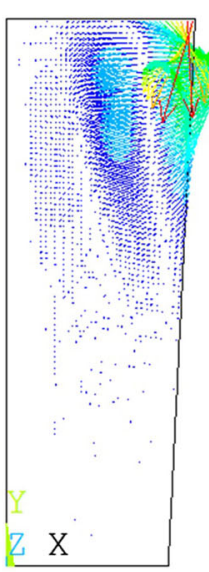

$.278 E-07$

048973

097947

.14692

.195893

.244867

.29384

. 342813

.391787

.44076

$\mathrm{T} / 4$

$\mathrm{T} / 2$

$3 T / 4$

T

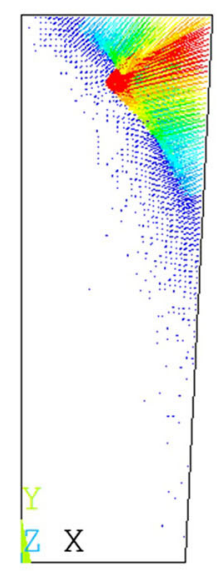

$\mathrm{T} / 4$

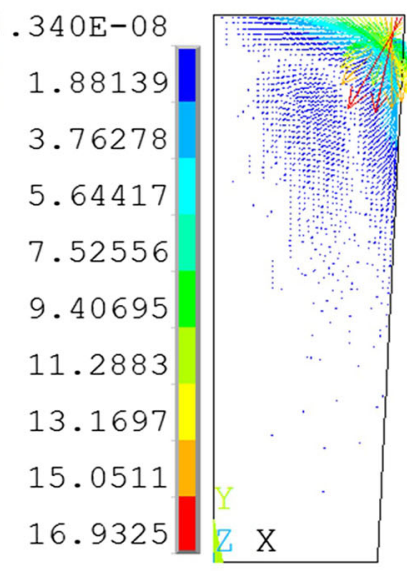

$\mathrm{T} / 2$

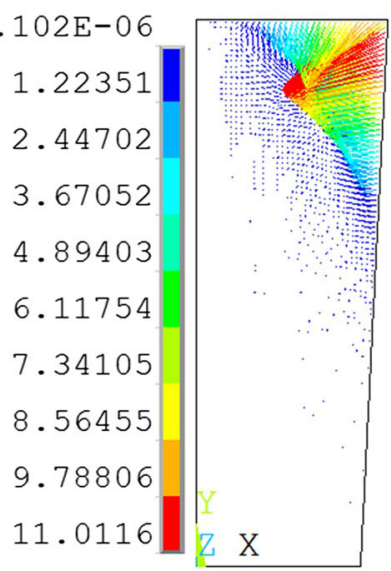

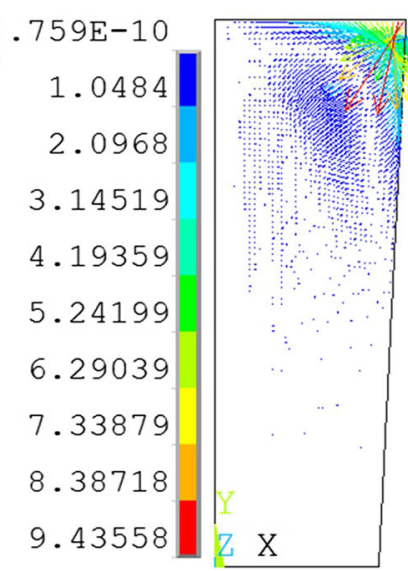

525E-07 .314605 .629211

.943816

1. 25842

1. 57303

1.88763

2. 20224

2. 51684

2. 83145

Fig. 8 Distribution of electromagnetic force: a SPMO; b HPMO; c CPMO 


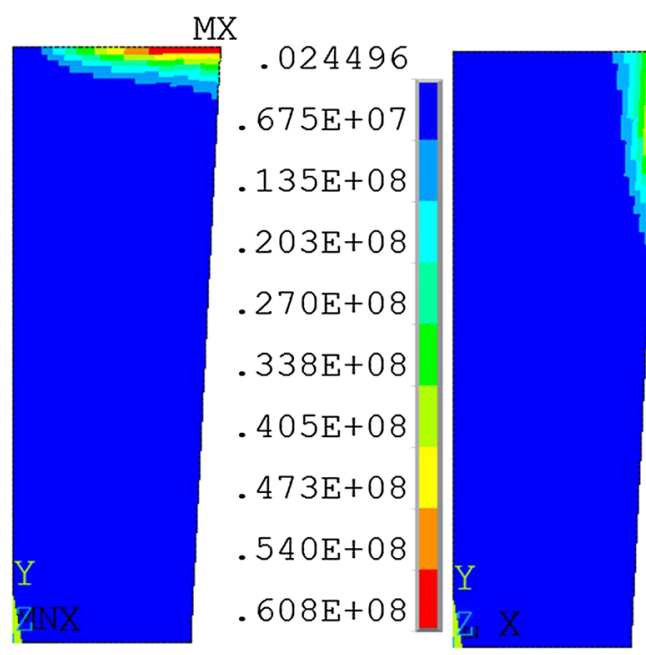

(a)

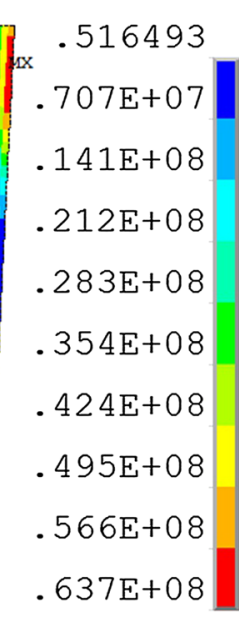

(b)

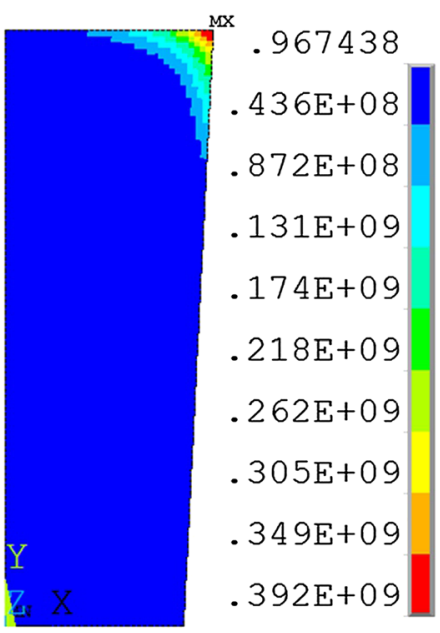

(c)

Fig. 9 Distribution of Joule heat at T/2: a SPMO; b HPMO; c CPMO

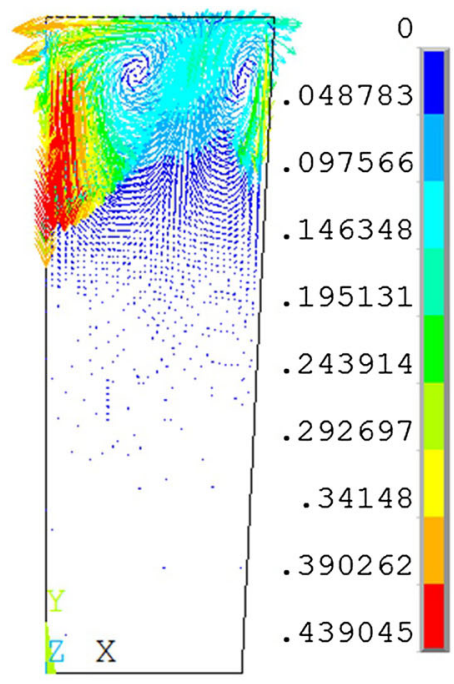

(a)

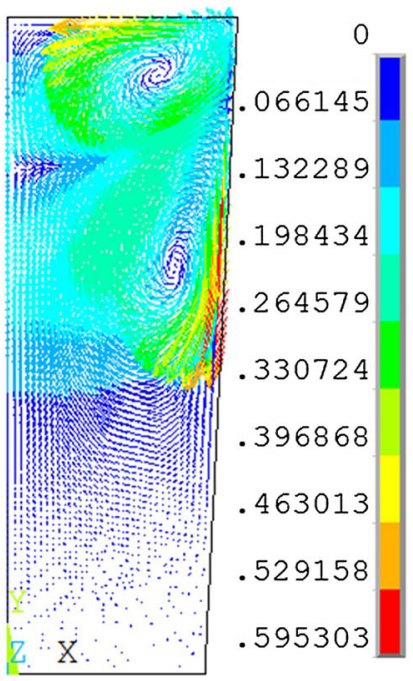

(b)

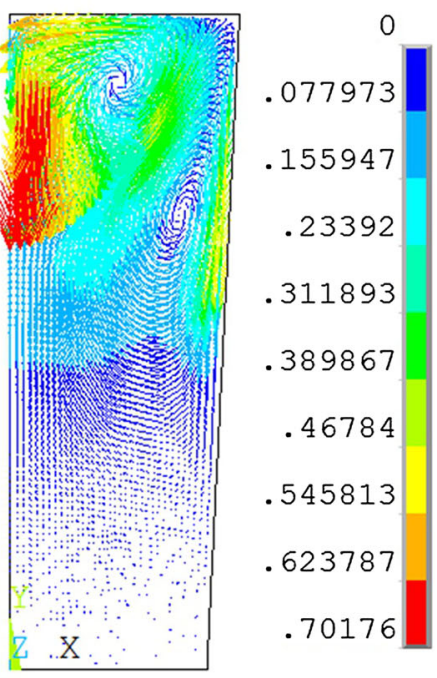

(c)

Fig. 10 Distribution of flow field: a SPMO; b HPMO; c CPMO

The size and distribution of shrinkage cavity differed also from PMO coil configuration. SPMO and CPMO reduced the depth of shrinkage cavity. The shrinkage cavity in HPMO ingot was similar to untreated ingot.

\section{Discussion}

\subsection{Formation of Equiaxed Zone}

The electromagnetic force in SPMO treated melt was concentrated in the skin zone on the top surface. Because of heterogeneous nucleation on the wall, more grains nucleated at the mold wall than near top surface. SPMOaffected region had limited numbers of grains drifted into the interior of melt. The regions affected by electromagnetic force in HPMO and CPMO cases were larger than that in SPMO. The value of electromagnetic force in the CPMO melt was over five times as large as in the HPMO. The direction of electromagnetic force in CPMO also favored the nucleus drifting. Thus, CPMO ingot had the largest volume fraction of equiaxed grains. 

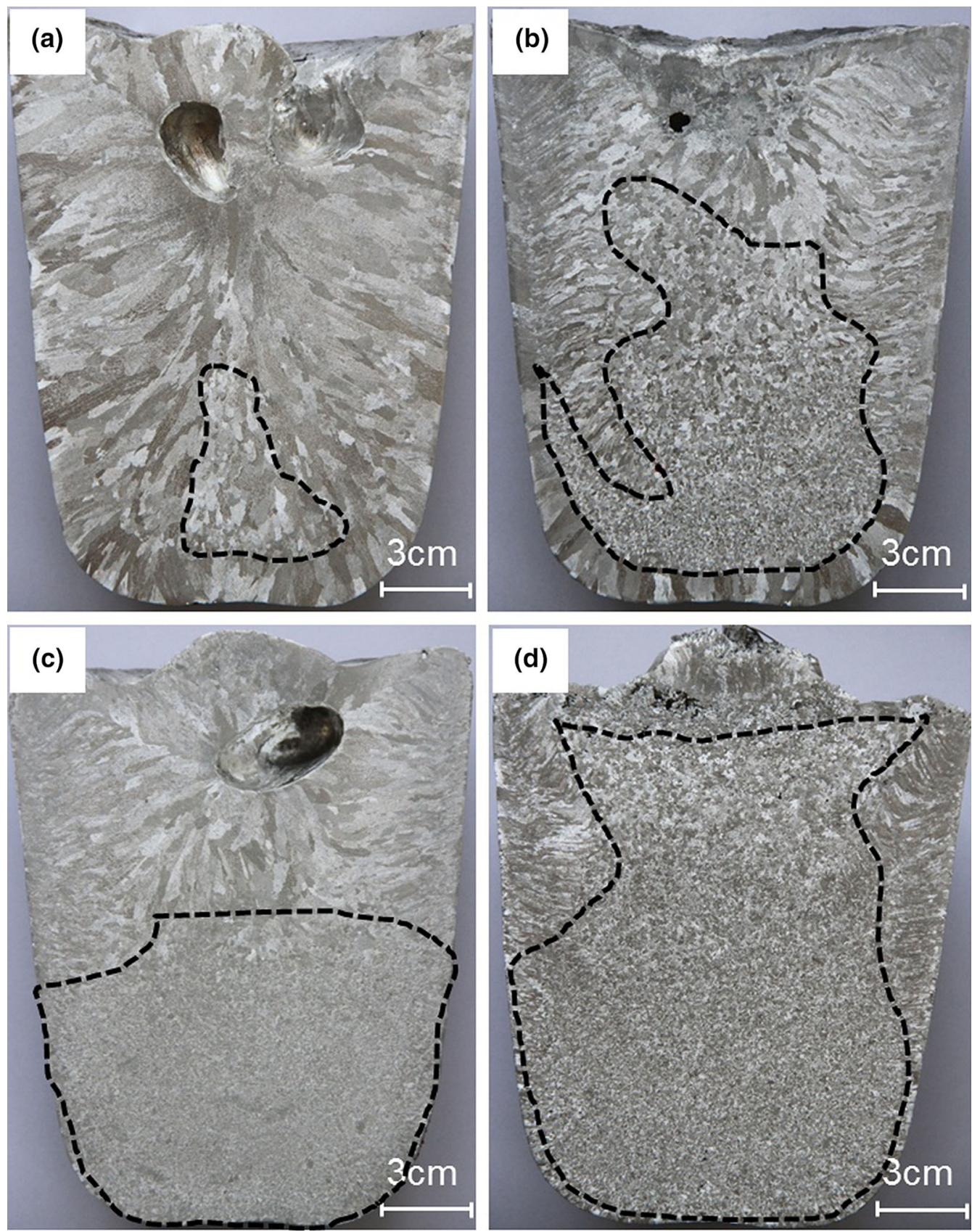

Fig. 11 Solidification structure of Al ingots: a untreated; b with SPMO; $\mathbf{c}$ with HPMO; d with CPMO. The dotted line circle marked the equiaxed zone

Table 2 Equiaxed crystal ratio and size of equiaxed grain of $\mathrm{Al}$ ingots

\begin{tabular}{lllll}
\hline & Untreated & SPMO & HPMO & CPMO \\
\hline Equiaxed crystal ratio & $2.5 \%$ & $37.13 \%$ & $41.46 \%$ & $81.3 \%$ \\
Size of equiaxed grain $(\mu \mathrm{m})$ & 1063 & 377 & 233 & 240 \\
\hline
\end{tabular}

\subsection{Size of Equiaxed Grains}

Although HPMO ingot had the smaller volume fraction of equiaxed zone, the size of equiaxed grains in HPMO ingot was similar to that of CPMO in lower part of the ingot.
This may be attributed to the heat transfer in the melt. Joule heat produced by PMO raised temperature at some locations. This heat prolonged solidification time, allowing sufficient time for grains drifting to refine solidification structure. Therefore, although a larger equiaxed zone was 

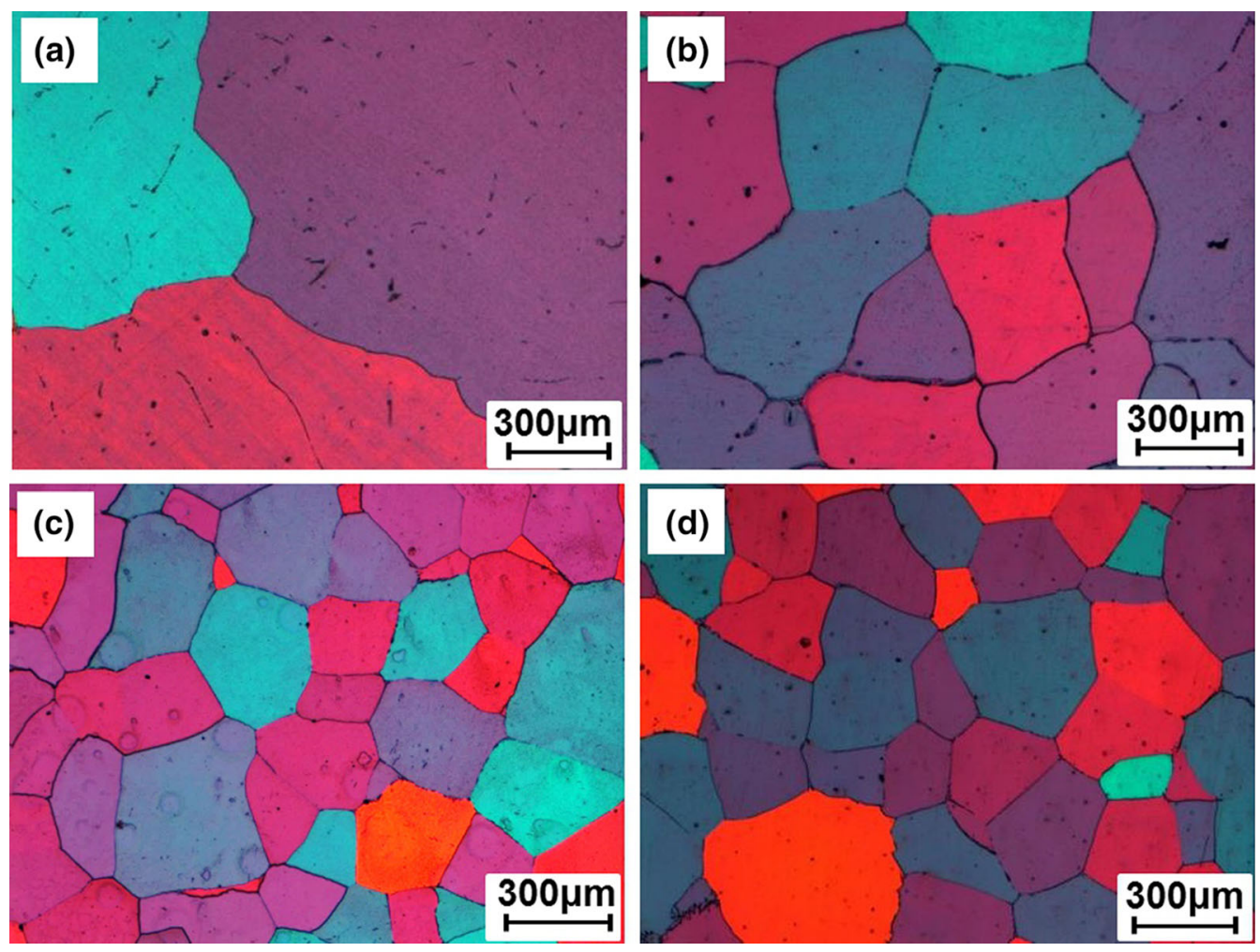

Fig. 12 Local solidification structure of Al samples: a untreated; b with SPMO; $\mathbf{c}$ with HPMO; d with CPMO. Micrographs taken at the lower half and center axil the ingots. Sample location can be found in Fig. 6

found in CPMO ingot, the grain growth induced by high temperature rendered. CPMO and HPMO had similar sized equiaxed grains.

\subsection{Columnar Grains in the Bottom of Ingots}

Larger electromagnetic force and available direction in HPMO and CPMO treated melt would drive more drifted grains faster to the bottom. That is to say, the drifted grains hindered the growth of columnar gains. Moreover, two vortexes near to the top surface in the SPMO treated melt would take free grains back to the top surface easily and smaller velocity speed the nuclei down. However, two vortexes approach to the interior of the HPMO and CPMO treated melt and larger velocity would speed the nuclei up to lower part. This is another reason for no columnar grains being founded at the bottom of the HPMO and CPMO treated ingots.

\subsection{Industrial Application}

In manufacture of industrial scale ingot, the hot-top of ingots is always removed. The solidification structure of lower part is the final product for production. The shrinkage cavity concentrated at the hot-top and cannot affect the ingot use. Our studies demonstrated that HPMO and
CPMO can produce similar refined grain size. In industrial application, HPMO coil could be embedded in the hot-top of mold and had no effect for pouring. The installation of CPMO coil may limit the method for pouring. The CPMO coil is, for example, inconvenient and unsafe when top pouring is used. SPMO had similar problem of CPMO and less refined effect. As a result, HPMO is more suitable than CPMO for large ingots production.

\section{Conclusions}

1. PMO produced equiaxed grain sized from $\mathrm{mm}$ scale to hundreds micrometer scale and increased the volume fraction of equiaxed grains by more than 10 times.

2. The coil design of PMO governed the distribution of electromagnetic field, flow field and solidification structure.

3. HPMO and CPMO had similar refinement effect. Both were better than SPMO.

4. The coil design of PMO influenced the texture of columnar growth.

5. HPMO is most suitable for industrial application.

Acknowledgements This work was financially supported by the National Natural Science Foundation of China (No. 51704210), the 
National Key Research and Development Program of China (No. 2017YFB0701802) and the Natural Science Foundation of Hebei Province (No. E2017105016). Part of work was undertaken in the National High Magnetic Field Laboratory, which is supported by US NSF DMR-1157490 and the State of Florida.

\section{References}

[1] O. Atsumi, Metal Solidification, Y.B. Tang, Z.D. Zhang, Mechanical Industry Press, Beijing, 1983, pp. 113-115

[2] H.Q. Yu, H.M. Gao, S.H. Zhang, W. Lin, Acta Metall. Sin. (Engl. Lett.) 22, 461 (2009)

[3] L. Chao, S.S. Jiang, K.F. Zhang, Z.P. Zhao, Acta Metall. Sin. (Engl. Lett.) 25, 153 (2012)

[4] J.M. Li, S. Li, J. Li, Scripta. Metal. Mater. 31, 1691 (1994)

[5] M. Nakada, Y. Shiohara, M.C. Flemings, ISIJ Int. 30, 27 (1990)

[6] C.Y. Ban, J.Z. Cui, Q.X. Ba, Acta Metall. Sin. (Engl. Lett.) 15, 380 (2002)

[7] Q.J. Zhai, Y.Y. Gong, Y.L. Gao, A Novel pulsed magneto oscillation method and device for solidification refinement, China Patent, No. ZL. 200510030739.8, 2007

[8] Y.Y. Gong, J. Luo, J.X. Jing, Q.J. Zhai, Mater. Sci. Eng., A 497, $147(2008)$
[9] Y.Y. Gong, Fundamental research on refinement of solidification structure of metals under pulse magneto oscillation, $\mathrm{Ph}$. D. Thesis, Shanghai University 2008. (in Chinese)

[10] N. Pei, Y.Y. Gong, R.X. Li, Q.J. Zhai, China Foundry 1, 112 (2011)

[11] Z.X. Yin, Y.Y. Gong, B. Li, Q.J. Zhai, J. Mater. Process. Technol. 212, 2629 (2012)

[12] B. Li, Z.X. Yin, Y.Y. Gong, Q.J. Zhai, China Foundry 8, 172 (2011)

[13] D. Liang, Z.Y. Liang, Q.J. Zhai, Mater. Lett. 130, 48 (2014)

[14] D. Liang, Z.Y. Liang, J. Sun, Q.J. Zhai, China Foundry 12, 48 (2015)

[15] I. Edry, V. Erukhimovitch, A. Shoihet, J. Mater. Sci. 48, 8438 (2013)

[16] I. Edry, T. Mordechai, N. Frage, Metall. Mater. Trans. A 47, 1 (2016)

[17] I. Edry, N. Frage, S. Hayun, Mater. Lett. 182, 118 (2016)

[18] J. Sun, C. Sheng, D.P. Wang, J. Zhao, Q.J. Zhai, China Foundry 15, 1 (2018)

[19] J. Zhao, Y.F. Cheng, K. Han, X.Z. Zhang, Q.J. Zhai, J. Mater. Process. Technol. 229, 286 (2016)

[20] X.L. Liao, Q.J. Zhai, J. Luo, Acta Mater. 55, 3103 (2007) 